\title{
Methamphetamine mimics the neurochemical profile of aging in rats and impairs recognition memory
}

Pedro Meloa, b, , , Ana Magalhães ${ }^{a}$, Cecília J. Alves ${ }^{b, c}$, Maria Amélia Tavares ${ }^{d}$, Liliana de Sousa ${ }^{e}$, Teresa

Summavielle ${ }^{a, b}$, Pedro Moradas-Ferreira ${ }^{a, e}$

a IBMC-Instituto de Biologia Molecular e Celular, Universidade do Porto, Porto, Portugal

b ESTSP-Escola Superior de Tecnologia da Saúde do Porto, Instituto Politécnico do Porto, Vila Nova de Gaia, Portugal

c INEB-Instituto Nacional de Engenharia Biomédica, Universidade do Porto, Porto, Portugal

d FMUP-Faculdade de Medicina, Universidade do Porto, Porto, Portugal

e ICBAS-Instituto de Ciências Biomédicas de Abel Salazar, Universidade do Porto, Porto, Portugal

\begin{abstract}
Brain neurochemistry and cognition performance are thought to decline with age. Accumulating data indicate that similar events occur after prolonged methamphetamine (MA) exposure. Using the rat as a model, the present study was designed to uncover common alteration patterns in brain neurochemistry and memory performance between aging and prolonged MA exposure. To this end, animals were treated with a chronic binge MA administration paradigm (20 mg/kg/day from postnatal day 91 to 100). Three-age control groups received isovolumetric saline treatment and were tested at the MA age-matched period, and at 12 and 20 months. We observed that both MA and aged animals presented a long, but not short, time impairment in novelty preference and an increased anxiety-like behavior. Neurochemical analysis indicated similar MAand age-related impairments in dopamine, serotonin and metabolites in the striatum, prefrontal cortex and hippocampus. Thus, the present data illustrate that MA may be used to mimic age-related effects on neurotransmitter systems and advocate MA treatment as a feasible animal model to study neuronal processes associated with aging.
\end{abstract}

\section{Keywords}

Methamphetamine; Accelerated aging; Neurochemistry; Recognition memory

\section{Introduction}

Methamphetamine (MA) is a highly addictive psychostimulant. Abuse of MA has increased dramatically to epidemic proportions worldwide and is an escalating public health issue (Cretzmeyer et al., 2003). MA abuse is associated with frontostriatal neurotoxicity (Salo et al., 2009), with morphometric alteration in brain areas such as hippocampus and cortex (Eisch et al., 1998 and Harvey et al., 2000), together with cognitive and decision-making impairments (Kalechstein et al., 2003, Nordahl et al., 2003, Scott et al., 2007 and Simon et al., 2004). Despite the publication of numerous studies in rodent models, knowledge of the types and the duration of behavioral alterations resulting from extended administration of MA remain limited (Marshall et al., 2007, Nagai et al., 2007 and Schroder et al., 2003). Although a number of studies report particular cognitive impairments in subjects with a history of amphetamine abuse (Meredith et al., 2005), distinguishing drug effects from other genetic and environmental conditions of these individuals is a complex task. In rodents, MA decreases various indices of dopamine terminal integrity in the striatum as the preferential target (Hotchkiss and Gibb, 1980 and Ricaurte et al., 1982). The molecular mechanisms of MA toxicity are 
precipitated by its rapid entry into the CNS, followed by its influx into monoaminergic vesicles. This leads to the displacement of monoamines into the cytoplasm and their release into the synaptic cleft, inducing an extracellular increase of dopamine (DA) (Melega et al., 1995 and Nash and Yamamoto, 1992) and a redistribution of cytosolic DA (Cubells et al., 1994 and Jones et al., 1998). MA triggers DA release from the cytosol to the extracellular space by means of reverse transport through DA transporters (DAT). Vesicular monoamine transporter $2\left(\mathrm{VMAT}_{2}\right)$ also plays an important role in the MA-induced toxicity. Studies using $\mathrm{VMAT}_{2}$ knockout mice showed a higher rate of manifestations of MA neurotoxicity compared with wild type mice (Fumagalli et al., 1999). Oxidation of DA produces $\mathrm{H}_{2} \mathrm{O}_{2}$ via monoamino oxidase A (MAO-A) (Capela et al., 2009 and Graham, 1978) leading to a decrease number of DATs (Fleckenstein et al., 1997 and Metzger et al., 2000). Prolonged access to MA induces substantial nerve terminal degeneration within dopaminergic neurons (Ricaurte et al., 1983a and Ricaurte et al., 1983b), together with depletions in the majority of DA-related markers (Hotchkiss and Gibb, 1980 and Koda and Gibb, 1973). Reduction in the extracellular content of DA and metabolites has been demonstrated following MA exposure (Robinson et al., 1990). Even early developmental exposure to MA has been correlated with long-term dopaminergic deficits (Bubenikova-Valesova et al., 2009). To a lesser extent, repeated MA exposure also affects serotonergic terminals. The drug may cause a reduction in the concentration of serotonin (5HT) and its respective metabolites, a decrease in the number of $5 \mathrm{HT}$ transporter binding sites, and a reduction in the activity of tryptophan hydroxylase (Ricaurte et al., 1980 and Wagner et al., 1980a).

The senescence process is characterized by a time-dependent functional decline of both tissues and organ systems, which is related to biochemical, physiological, genetic and anatomical degeneration (Carnes et al., 2008 and Troen, 2003). It is known that drug abusers present several signs of accelerated aging. Indeed, age-related development and a more rapid evolution of various co-morbidities are observed in chronic consumers of drugs of abuse (Carvalho, 2009). Long-term abuse of psychostimulants is linked to premature atherosclerosis, ventricular hypertrophy, cardiomyopathies (Dowling et al., 2008) and kidney failure (Vupputuri et al., 2004). Moreover, evidence suggests that memory dysfunction is a consequence of aging (for review see Erickson and Barnes, 2003). Alterations in the neurochemistry of the different brain regions may offer valid cues to investigate the processes implicated in memory loss (Luine et al., 1990).

It has long been known that exposure to MA impairs cognition (Belcher et al., 2006, Belcher et al., 2008,Bisagno et al., 2002, Schroder et al., 2003 and White et al., 2009). However, the acute high doses delivered in the majority of studies hardly mimic the human drug abuse pattern. A recent report showed that extended daily access to MA (6 h) impaired novel object recognition memory, but the effect was not observed after only short periods (1 or $2 \mathrm{~h}$ ) of exposure (Rogers et al., 2008).

When working with rodent models, memory components can be evaluated using the object recognition (OR) task (Ennaceur et al., 2005). This task measures recognition memory based on spontaneous exploration behavior and innate tendency to novelty preference. The OR task has several advantages in terms of relating the neurochemical profile to memory performance: First, the task does not use a positive (e.g., food/water rewards) or negative (e.g., electrical shocks) reinforcement, which may interfere with factors such as anxiety levels. Second, animals are not required to learn contingency rules to perform the tasks. Third, stress-related confounding variables can be minimized since animals do not encounter stressful circumstances, such as forced swimming in the Morris water maze (Ennaceur et al., 1997).

Since the nigrostriatal DA system is impaired after extended MA exposure and during the senescence process, the present study was designed to uncover common alteration patterns in brain neurochemistry and memory performance between chronological aging and prolonged MA exposure. Moreover, we endeavor to contribute to the development of a model that mimics the age-related effects to the neurotransmitter systems and to the recognition memory of the rat. 


\section{Materials and methods}

\subsection{Experimental design and drug administration}

Forty-four adult male Wistar rats from Charles Rivers (Barcelona, Spain) were used. All experiments were performed in a licensed environmental controlled room (respecting the Guidelines of the European Union Council 86/609/EU and Portuguese Law Decreto-Lei No 1005/92) at the Instituto de Biologia Molecular e Celular (IBMC), Universidade do Porto. Food and water were freely available. (+)-MA hydrochloride (SigmaAldrich, St. Louis, MO, USA) was dissolved in saline ( $\mathrm{NaCl}, 0.9 \%$ ) and administrated in a volume of $1 \mathrm{~mL} / \mathrm{kg}$ injected intraperitoneally. Changes in body weight were registered daily. Each group consisted of at least 6 animals. Rats were assigned to their respective groups at postnatal day (PND) 60 (2 animals of the same experimental group per cage). Treated rats received $5 \mathrm{mg} / \mathrm{kg}$ of MA every $2 \mathrm{~h}$ for a period of $6 \mathrm{~h}$ (i.e., 4 injections) each day between PND 91 and 100. Between injection, stereotypies were observed, nevertheless, they were absent at the time of all behavioral measurements. The three groups (MA agematched, and chronologically aged 12 months and 20 months rats) received an isovolumetric dose of saline following the same age and injection pattern to equal stress induction produced in the administration of MA. The dose chosen corresponded well with previous work by our group (Melo et al., 2010), and by the Vorhees group where they investigated the effect of MA exposure, comprising 4 doses per day of $5 \mathrm{mg} / \mathrm{kg}$ of MA at $2 \mathrm{~h}$ intervals, from PND 51 to 60 after which long-term spatial and sequential learning deficits were observed (Vorhees et al., 2005).

\subsection{Habituation}

On the first day after the last administration of MA, animals were exposed for the first time to the experimental room and the test apparatus. The first session served as an anxiety test, which was followed by four other habituation trials. An object was placed in the center of the arena. On habituation days $1-5$, each rat was placed in the arena and the behavior was recorded for $10 \mathrm{~min}$. The MA age-matched control group followed the same protocol. The two groups of aged animals were tested at the respective time point (12 or 20 months) following the same protocol.

\subsection{Object recognition task}

The OR task used in this study (Fig. 1) was adapted from previous work (Ennaceur et al., 2005). All trials were performed in the dark period and in an open field arena (width $\times$ length $\times$ height $=60 \mathrm{~cm} \times 80 \mathrm{~cm} \times 40 \mathrm{~cm}$ ) made of gray PVC. Intensity of light $(100 \mathrm{~lx})$ was carefully controlled to illuminate the floor of the chamber evenly. The objects to be discriminated were made of a biologically neutral material, such as plastic (cube with $6 \mathrm{~cm} \times 6 \mathrm{~cm} \times 6 \mathrm{~cm}$ ), metal (pyramid with $10 \mathrm{~cm}$ in the base and $10 \mathrm{~cm}$ of height) or glass (cylinder with $6 \mathrm{~cm}$ of diameter and $10 \mathrm{~cm}$ of height), and fitted to the surface of the arena so that the animals could not move them around. Between all trials the surface of the arena and objects was cleansed with neutral soap (Extran, Merck, Darmstadt, Germany). All trials were videotaped with a SONY DCR-SR290E camcorder, suspended above the test arena, and analyzed afterward with the software package Observer XT 7.0 (Noldus Information Technology, Wageningen, The Netherlands). 


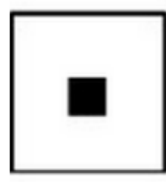

10 mis babituation 1st-5th day
Object recognition $15 \mathrm{~min}$ delay

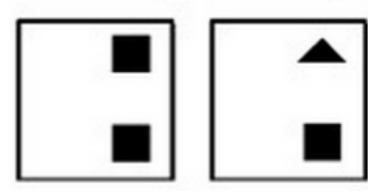

3 min sumple trial 3 min retention trial $6^{\text {th }}$ day
Object recognition 24 hour delay

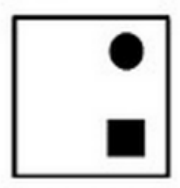

3 min retention trial

7th day

Fig. 1.

Schematic representation of the object recognition task. The 1 st exposure to the test apparatus and to the object served as an anxiety test (frequency of approaches). The $2 \mathrm{nd}-5$ th exposures to the apparatus and object served as an habituation process. In the object recognition trials, the 6th exposure to the apparatus and the objects served as a sample trial and the following two exposures served as the short time retention trial ( $15 \mathrm{~min}-6$ th day) and long time retention ( $24 \mathrm{~h}-7$ th day).

The OR test was performed on the sixth and seventh day after the beginning of the habituation process. It consisted of a first session of a 3-min sample trial and two different retention trials. The short retention time was considered $15 \mathrm{~min}$ and the long retention time $24 \mathrm{~h}$. During the sample trial, two identical objects were placed at one end of the open field arena, $10 \mathrm{~cm}$ away from the sidewall. The total amount of time that the animals spent exploring the objects during the $3 \mathrm{~min}$ was recorded. In each of the two different retention trials, a new object replaced one of the familiar objects. The time spent by the animal exploring the familiar and the new object was recorded for a 3 min duration.

\subsection{Behavioral measurements}

The basic measures were the time spent by the rats exploring objects during the first habituation trial, during the sample phase and during the choice phase. Exploration of an object was defined as directing the nose to the object at a distance of approximately $2 \mathrm{~cm}$ and/or touching it with the nose. Conversely, turning around or sitting on the object was not considered as exploratory behavior. Considering the overall exploration of objects in the different trials, the following measurements were analyzed: the frequency of approaches, which refers to the number of times a rat approached each object; the total object exploration, which refers to the amount of time spent by a rat exploring the objects; the object exploration, which refers to the amount of time spent by a rat exploring each object; the object exploration frequency, which refers to the number of times a rat explored each object. The discrimination index measures the response of animals in exploring novelty and familiarity and is calculated by the product of the difference (subtraction) between time and frequency of exploration of new and familiar objects. A value that is significantly above zero describes animals exploring more the novel than the familiar object. A value that is significantly below zero describes animals exploring more the familiar than the novel object.

\subsection{Neurochemistry: monoamines and metabolites}

The day after completion of behavioral studies, six animals from each experimental group were used for neurochemical analysis. Rats were sacrificed by decapitation and the brains rapidly dissected on ice under dim light and stored at $-70{ }^{\circ} \mathrm{C}$ until determination. The striatum, prefrontal cortex and hippocampus were used in this study. The different brain regions were ultrasonicated (Sonifier W-250, Branson Ultrasonics, Danbury, CT) in $200 \mu \mathrm{L}$ of ice-cold $0.2 \mathrm{M}$ perchloric acid. The homogenate was centrifuged at $13,000 \times$ gduring $15 \mathrm{~min}$ at $4{ }^{\circ} \mathrm{C}$ and the supernatant was filtered thought a $0.2 \mu \mathrm{m}$ Nylon-66 microfilter. Aliquots of $50 \mu \mathrm{L}$ were analyzed by high-performance liquid chromatography (HPLC) with electrochemical detection (ECD) ( Ali et al., 1993 and Alves et al., 2009). The HPLC system used is composed of an LC234 
auto-injector, an LC307 delivery pump, an LC142 electro-detector, and a 712 HPLC system controller data version 1.30 management software (all supplied by Gilson Medical Electronics HPLC System, Middleton, $\mathrm{WI}$ ). The mobile phase consisted of $70 \mathrm{mM}$ of potassium phosphate monobasic buffer ( $\mathrm{pH}$ adjusted to 3.0 by adding phosphoric acid), $1 \mathrm{mM} 1$-heptanosulfonic acid, $107.5 \mu \mathrm{M}$ sodium EDTA, and 10\% (v/v) of methanol. The flow was maintained at $0.8 \mathrm{~mL} / \mathrm{min}$. Chromatographic separation was achieved using a supercosil LC $(7.5 \mathrm{~cm} \times 4.6 \mathrm{~cm}, 3 \mu \mathrm{m})$ reverse phase column. Detection was achieved with an electrochemical detector equipped with a glassy carbon-working electrode set at $+0.75 \mathrm{~V}$ and quantification was by external standard method. Protein content was determined using the Bio-Rad Bradford protein assay. Results were expressed in $\mathrm{ng} / \mathrm{mg}$ of protein. Turnover (TO) ratios were calculated as measure of activity (metabolites/monoamine).

\subsection{Data analysis}

Anxiety responses were assessed by analyzing the behavioral data collected during the first exposure to the object with a one-way ANOVA (group) followed, when appropriate, by Tukey's post hoc test. The delay effect on object recognition ability was assessed by ANOVA with repeated measures for delay. Mean differences among group were tested for significance by one-way ANOVA followed, when appropriate, by Tukey's post hoc test. A paired Student $t$-test was also performed within group comparisons to demonstrate the presence of novelty or familiarity preference by comparing measurement on time spent in exploration of new object to that spent in exploration of familiar object. Neurochemical data were analyzed with a one-way ANOVA (group), followed, when appropriate, by Tukey's post hoc test. To facilitate data comprehension across the result presentation when results refer to differences from the control group the $\S$ sign is used, when results refer to differences from the MA group the \# sign is used, and when results refer to differences from 12 mounts the + sign is used. All results were analyzed at a significance level of $5 \%$, using the software SPSS version 17.0 for Windows.

\section{Results}

\subsection{Recognition memory impairment in both MA and aged animals}

Repeated measures ANOVA for delay in terms of total time spent exploring the objects revealed a main effect of group $[F(3)=8.82, p<0.01]$, and a significant interaction between group and delay $[F(3)=3.37, p<0.05]$. The post hoc comparisons revealed that animals from MA group explored more the objects at the delay 15 min when compared with the control group, the 12 months group and the 20 months group ( $p<0.001$ for all groups). Concerning the $24 \mathrm{~h}$ delay the animals from MA group explored more the objects when compared with the 12 months group and the 20 months group ( $p<0.05$ for both groups). The control group explored more the objects in the $24 \mathrm{~h}$ delay when compared with both the 12 months and 20 months groups $(p<0.05)$.

Repeated measures ANOVA for the time spent exploring the new object revealed a significant interaction between group and delay $[F(3)=5.69, p<0.01]$ but not a significant effect for delay. The same analysis for the time spent exploring the familiar object showed no effect of delay and no significant interaction between group and delay ( Fig. 2). The one-way ANOVA used to evaluate differences among groups showed a main effect of group on the time spent exploring the new object $[F(3)=6.87, p<0.001]$ and on the time spent exploring the familiar object $[F(3)=7.03, p<0.05]$. In the 15 min delay, the post hoc comparisons revealed that animals from the MA group explored more, both new (control, 12 months and 20 months: $p<0.01$ ) and familiar (control $p<0.05,12$ months $p<0.001,20$ months $p<0.05$ ) objects, when compared with the other groups. In the $24 \mathrm{~h}$ delay, the post hoc comparisons revealed that control animals explored more the new 
object when compared with the group 12 months $(p<0.01)$; and the MA group explored more the familiar object when compared with the 12 months group $(p<0.05)$.

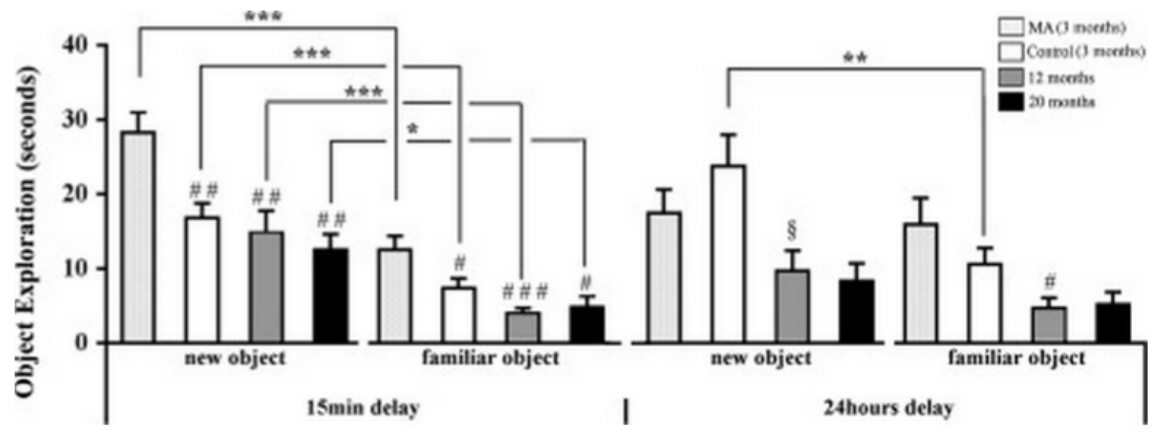

Fig. 2.

Exploration is increased in MA-treated animals and novelty preference is impaired in both MA-treated and aged animals. Effects of binge chronic administration of MA ( $20 \mathrm{mg} / \mathrm{kg} /$ day split in 4 doses from postnatal day 91 to 100$)$ and chronological aging (MA agematched, 12 or 20 months old) on the duration of the exploration of both new object and familiar object at $15 \mathrm{~min}$ delay and $24 \mathrm{~h}$ delay. Results are expressed as mean \pm S.E.M. of 6-14 animals per group. Different from MA: ${ }^{*} P<0.05 ;{ }^{\#} P<0.01 ;{ }^{\# \#} P<0.001$. Different from control: ${ }^{\S} P<0.05$. ${ }^{\star} P<0.05 ;{ }^{* \star} P<0.01 ;{ }^{* * \star} P<0.001$.

In the 15 min delay, the paired-sample $t$-test for within group comparisons revealed that all experimental groups preferentially (MA, control and 12 months $p<0.001 ; 20$ months $p<0.05$ ) explored the new object. At the longer delay, $24 \mathrm{~h}$, only the control group retained this behavior $(p<0.01)$.

Statistical analysis of the $24 \mathrm{~h}$ delay discriminatory index based on the duration and frequency of the object exploration (Fig. 3) revealed a significant effect of group $[F(3)=4.46, p<0.05]$ and $[F(3)=2.89, p<0.05]$, respectively. Indeed, post hoc analysis showed that the discrimination index of MA group was significantly impaired when compared with the control group ( $p<0.05$ for both duration and frequency). 

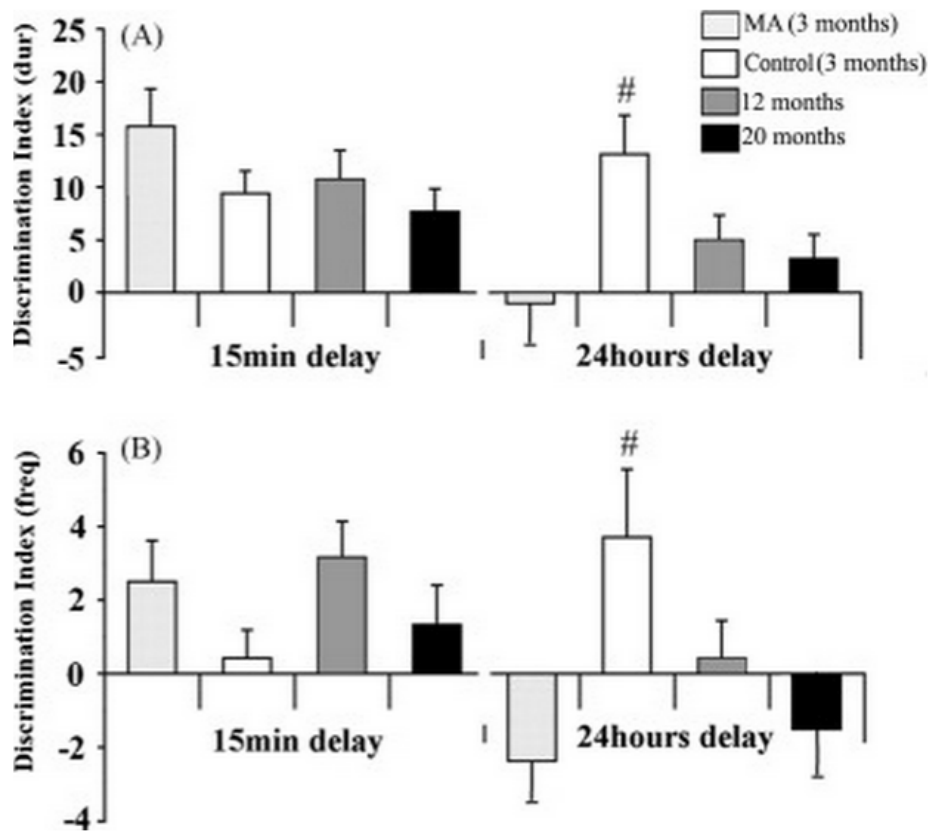

Fig. 3.

Impaired long-term discrimination index in MA-treated animals. Effects of binge chronic administration of MA (20 mg/kg/day split in 4 doses from postnatal day 91 to 100) and chronological aging (MA age-matched, 12 or 20 months old) on the discrimination index between the new and familiar object based on the duration $(A)$ and frequency $(B)$ of exploration (15 min and $24 \mathrm{~h}$ delay). Results are expressed as mean \pm S.E.M. of 6-14 animals per group. Different from MA: ${ }^{\#} P<0.05$.

\subsection{Increased anxiety in MA and aged animals}

The one-way ANOVA of the behavior data collected during the first exposure to the arena revealed a significant effect of group in the frequency of object approaches $[F(3)=17.29, p<0.001]$. Post hoc analysis showed that the control group presented the highest frequency of approaches, significantly differing from the MA $(p<0.05), 12$ months $(p<0.001)$ and 20 months $(p<0.001)$ groups. The MA group presented a significant increase in frequency of object approaches when compared with the 12 months group $(p<0.05)$. The data are presented in Fig. 4. The overall results point to an increased anxiety and neophobic behavior, which can be interpreted by the reduced interaction with the object, in both MA and aged animals.

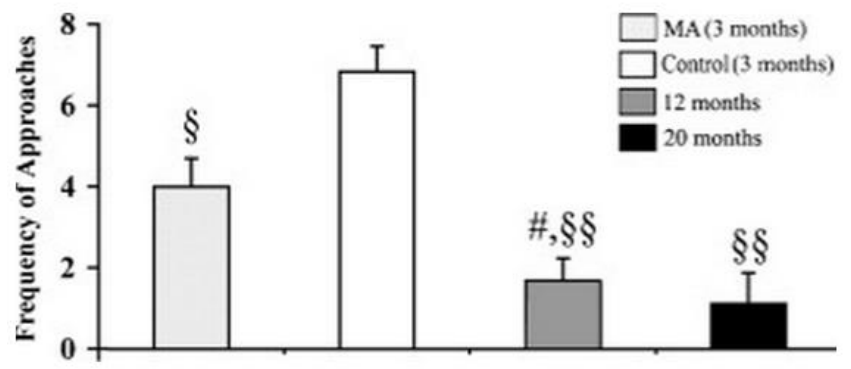

Fig. 4 .

Increased anxiogenic response in both MA-treated and aged animals in the first section of the habituation process. Effects of binge chronic administration of MA (20 mg/kg/day split in 4 doses from postnatal day 91 to 100$)$ and chronological aging (MA agematched, 12 or 20 months old) on the frequency of approaches to an object. Results are expressed as mean \pm S.E.M. of 6-11 animals per group. Different from MA: ${ }^{p} p<0.05$. Different from control: ${ }^{\S} p<0.05 ;{ }^{\S} p<0.01$. 


\subsection{Altered neurotransmission in both MA and aged animals}

To determine whether the present MA exposure paradigm fits the standard decline of neurochemical profile associated with aging, monoamines, metabolites and respective turnover ratios were measured. Results concerning DA and metabolites are presented in Fig. 5 and results concerning $5 \mathrm{HT}$ and 5hydroxyindoleacetic acid (5HIAA) are presented in Fig. 6. Data concerning activity levels are represented in Table 1 as TO ratios.
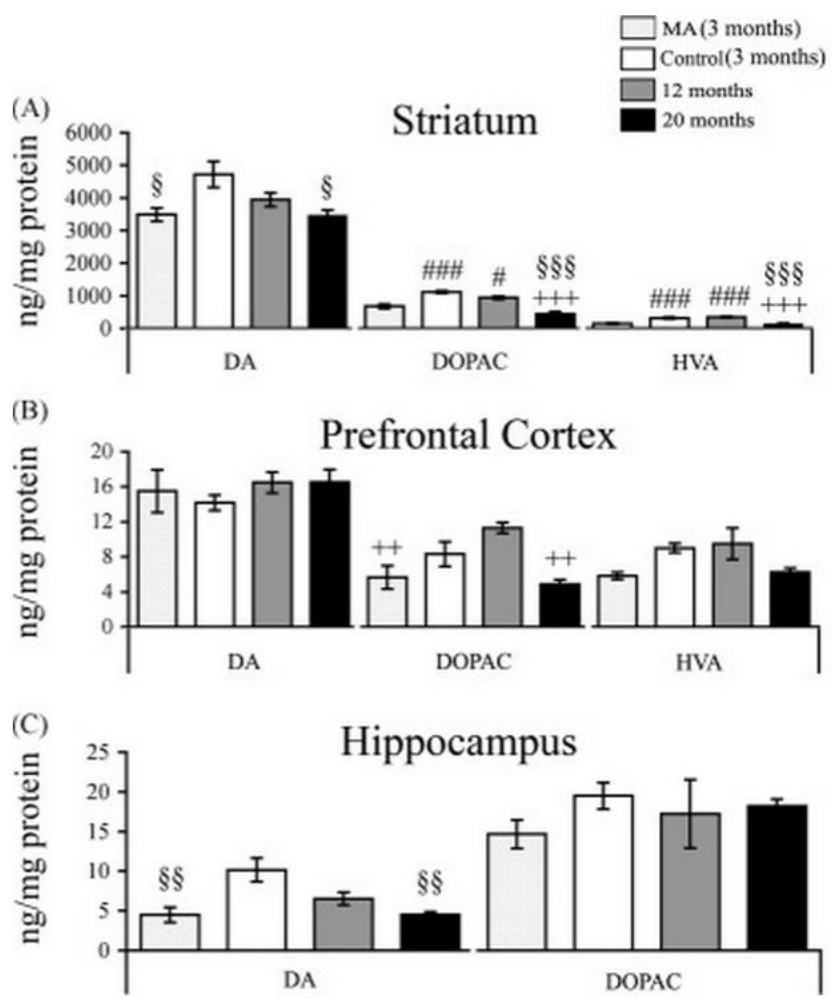

Fig. 5.

Levels of DA and metabolites in the striatum (A), prefrontal cortex (B) and hippocampus (C). Rats were treated with MA $(20 \mathrm{mg} / \mathrm{kg} /$ day split in 4 doses from postnatal day 91 to 100) or were chronologically aged (MA age-matched, 12 and 20 months old). Results are expressed as mean \pm S.E.M. of 6 per group. Different from MA: ${ }^{\prime} p<0.05$; $\#<0.001$. Different from control: $\S p<0.05 ; § \S p<0.01 ; \S \S \S<0.001$. Different from 12 months: ${ }^{++} P<0.01 ;{ }^{+++} P<0.001$. 

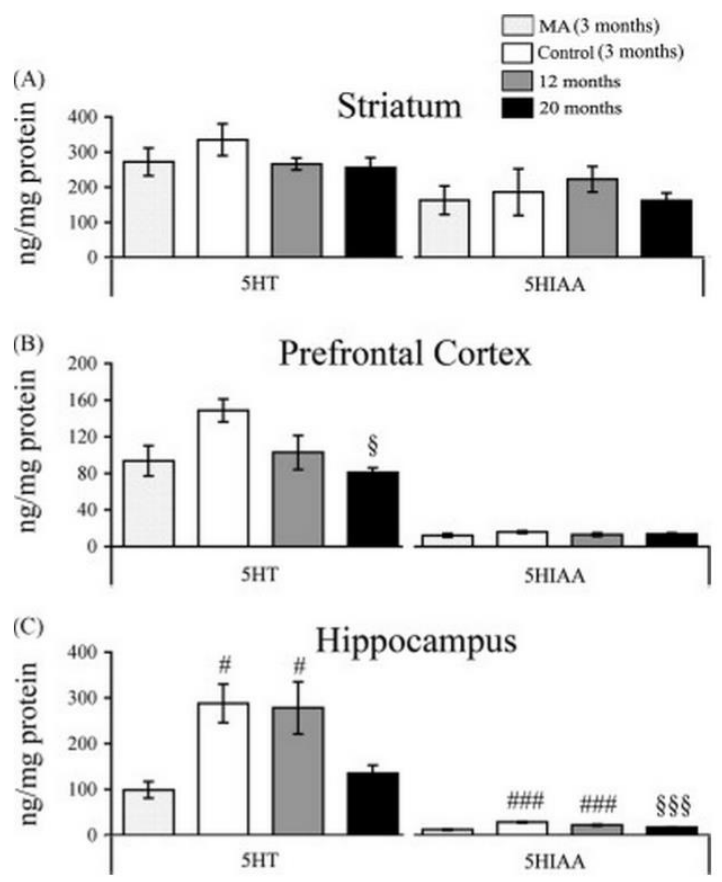

Fig. 6.

Levels of $5 \mathrm{HT}$ and metabolites in the striatum (A), prefrontal cortex (B) and hippocampus (C). Rats were treated with MA ( $20 \mathrm{mg} / \mathrm{kg} /$ day split in 4 doses from postnatal day 91 to 100 ) or were chronological aged (MA age-matched, 12 and 20 months old). Results are expressed as mean \pm S.E.M. of 6 per group. Different from MA: ${ }^{*} P<0.05$; ${ }^{m} P<0.001$. Different from control: $\S p<0.05 ; \S \S p<0.001$.

Table 1.

Turnover rates for DA, expressed as [DOPAC/DA; HVA/DA; (DOPAC + HVA)/DA], and for 5HT, expressed as [5HIAA/5HT] in the striatum, prefrontal cortex and hippocampus. Rats were treated with MA ( $20 \mathrm{mg} / \mathrm{kg} /$ day split in 4 doses from postnatal day 91 to 100 ), or were chronologically aged (MA age-matched, 12 and 20 months old).

\begin{tabular}{|c|c|c|c|c|}
\hline Turnover & Group & Striatum & Prefrontal cortex & Hippocampus \\
\hline \multirow[t]{4}{*}{ DOPAC/DA } & MA (3 months) & $0.19(0.01)$ & $0.39(0.78)$ & $3.82(0.80)$ \\
\hline & Control (3 months) & $0.24(0.02)$ & $0.60(0.11)$ & $2.04(0.21)$ \\
\hline & 12 months & $0.24(0.02)$ & $0.71(0.06)^{\#}$ & $3.44(1.48)$ \\
\hline & 20 months & $0.13(0.02)$ & $0.31(0.04)^{+}$ & $4.10(0.29)$ \\
\hline \multirow[t]{4}{*}{ HVA/DA } & MA (3 months) & $0.04(0.01)$ & $0.41(0.05)$ & - \\
\hline & Control (3 months) & $0.07(0.01)^{\#}$ & $0.64(0.05)$ & - \\
\hline & 12 months & $0.09(0.01)^{\# \#}$ & $0.58(0.12)$ & - \\
\hline & 20 months & $0.04(0.01)$ and & $0.40(0.05)$ & - \\
\hline \multirow[t]{4}{*}{$(\mathrm{DOPAC}+\mathrm{HVA}) / \mathrm{DA}$} & MA (3 months) & $0.23(0.02)$ & $0.81(0.11)$ & $3.82(0.80)$ \\
\hline & Control (3 months) & $0.31(0.02)$ & $1.24(0.15)$ & $2.04(0.21)$ \\
\hline & 12 months & $0.33(0.03)^{\#}$ & $1.29(0.14)$ & $3.44(1.48)$ \\
\hline & 20 months & $0.17(0.02)$ and & $0.70(0.07)$ and & $4.10(0.29)$ \\
\hline
\end{tabular}




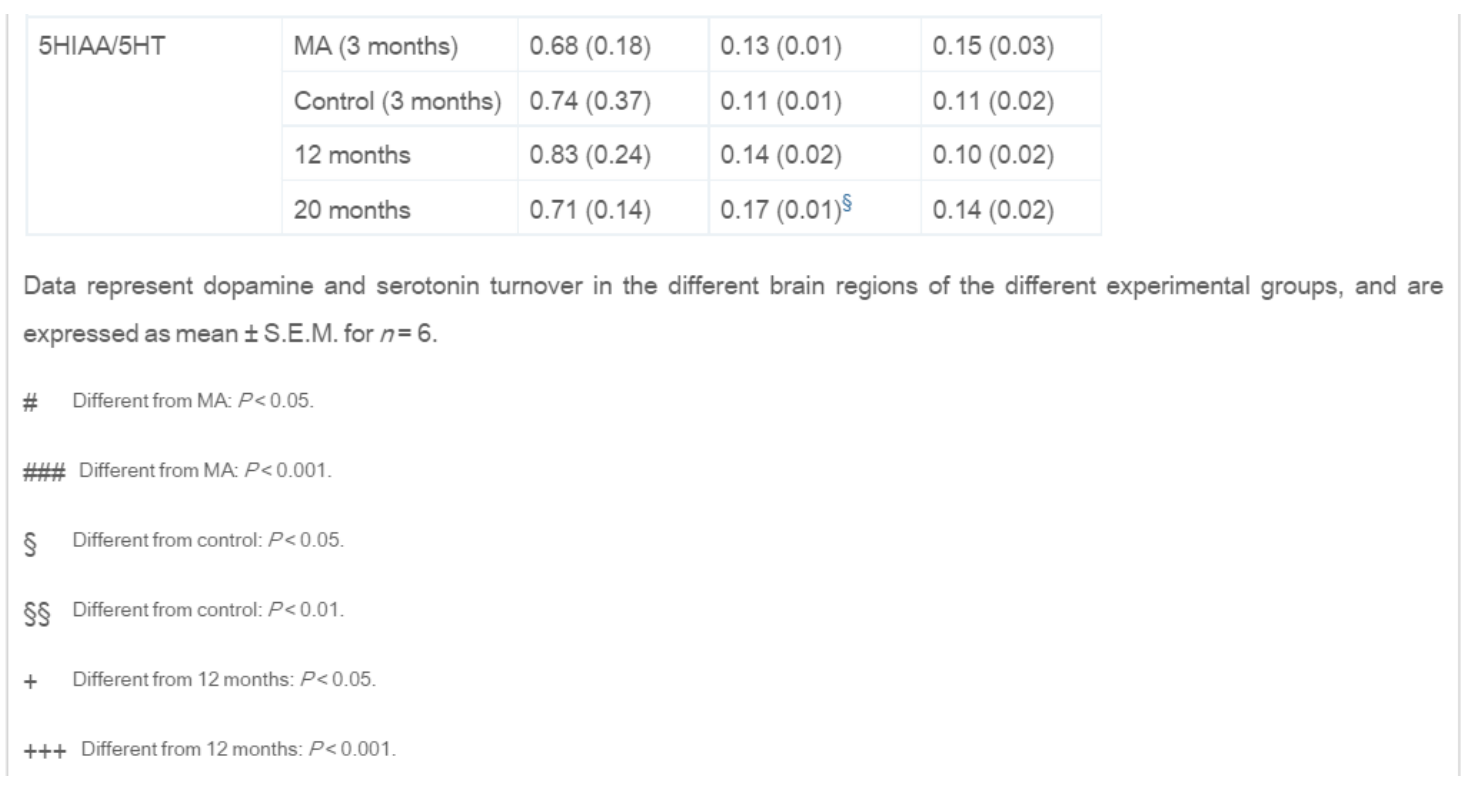

\subsubsection{Dopamine and metabolites}

Statistical analysis of DA, 3,4-dihydroxyphenylacetic acid (DOPAC) and homovanilic acid (HVA) contents in the striatum revealed a significant effect of group $[F(3)=5.08, p<0.01 ; F(3)=25.31, p<0.001 ; F(3)=22.98, p<0.001$; respectively]. In the striatum, the levels of DA and metabolites (DOPAC and HVA) were generally higher in the control group when compared with the respective aged-matched MA group and both aged rat groups (12 and 20 months). The DA levels were significantly reduced in the MA group when compared with the control group $(26 \%, p<0.05)$. In the 20 months group, the level of DA was significantly reduced when compared with the control group $(27 \%, p<0.05)$. The DOPAC level was significantly increased in the control group when compared with both the MA group $(40 \%, p<0.001)$ and the 20 months group $(60 \%, p<0.001)$. When compared with the 12 months group, both MA and 20 months groups presented reduced DOPAC levels (28\%, $p<0.05$ and $53 \%, p<0.001$, respectively). The HVA level was significantly higher in the control group when compared with both the MA group $(54 \%, p<0.001)$ and the 20 months group $(56 \%, p<0.001)$. When compared with the 12 months group, both MA and 20 months groups presented reduced HVA levels (approximately $58 \%, p<0.001$ for both). No other significant differences in DA and metabolites levels were found in the striatum ( Fig. 5A).

Statistical analysis of the DOPAC content in the prefrontal cortex revealed a significant effect of group $[F(3)=7.69, p<0.001]$. In the prefrontal cortex, the DOPAC level was significantly increased in the 12 months group when compared with both the MA group $(50 \%, p<0.01)$ and the 20 months group $(56 \%, p<0.01)$. No significant differences between groups were detected concerning DA or HVA levels ( Fig. $5 \mathrm{~B})$.

Statistical analysis of the DA content in the hippocampus revealed a significant effect of group $[F(3)=7.37, p<0.01]$. The DA level in the hippocampus was impaired in all studied groups when comparing with the control group. These differences reached significance in both the MA group $(56 \%, p<0.01)$ and 20 months group $(55 \%, p<0.01)$. Even though the impairment between the 12 months group and the control did not reach significance, a $35 \%$ decrease was observed. No significant differences were observed in the level of DOPAC when comparing the different studied groups. In the hippocampus, the level of HVA was below the detection level of the method ( Fig. 5C). 


\subsubsection{Serotonin and 5 HIAA}

In the striatum, the levels of 5HT and 5HIAA did not significantly differ between the different studied groups (Fig. 6A).

Statistical analysis of the $5 \mathrm{HT}$ content in the prefrontal cortex revealed a significant effect of group $[F(3)=4.34, p<0.05]$. The $5 \mathrm{HT}$ level in the prefrontal cortex of control animals presented the highest value, reaching a significant difference with the 20 months group ( $45 \%, p<0.05)$. The impairment of $37 \%$ observed in the MA group did not reach significance. In the prefrontal cortex, the level of 5HIAA was not significantly different between the studied groups ( Fig. 6B).

Statistical analysis of the serotonin and 5HIAA contents in the hippocampus revealed a significant effect of group $[F(3)=6.61, p<0.01 ; F(3)=20.58, p<0.001$; respectively]. In the hippocampus, an impaired level of $5 \mathrm{HT}$ was observed in the MA group when compared with both the control group $(65 \%, p<0.05)$ and the 12 months group $(64 \%, p<0.05)$. Concerning the 5HIAA values, the highest level was observed in the control group. The difference was significant when compared with both the MA group $(58 \%, p<0.001)$ and the 20 months group $(40 \%, p<0.001)$. Still considering 5HIAA contents, a significant impairment in the MA group when compared with 12 months group $(46 \%, p<0.001)$ was also observed ( Fig. $6 \mathrm{C}$ ).

\subsubsection{Turnover (TO) ratios}

The different DA TO ratios [DOPAC/DA, HVA/DA, and (DOPAC + HVA)/DA] and 5HT TO ratio (5HIAA/5HT) were calculated in the different brain regions (Table 1).

Statistical analysis of DA TO ratios in the striatum revealed a significant effect of group $[F(3)=7.66, p=<0.01 ; F(3)=15.45, p<0.001 ; F(3)=12.29, p<0.001$; respectively]. In the striatum, the highest HVA/DA ratios were observed in the control and in the 12 months groups. The impairment between the MA group and the control group, and between the 20 months and control group reached significance (both $43 \%, p<0.05$ ). In the MA and the 20 months groups, the HVA/DA ratios were significantly impaired when compared with the 12 months group (approximately 55\%, $p<0.001$ for both). Concerning $($ DOPAC $+\mathrm{HVA}) / \mathrm{DA}$, the 12 months group presented the highest value, even though not significantly differing from the control group. When compared with the 12 months group, both the MA and the 20 months groups displayed impairments ( $30 \%, p<0.001$ and $48 \%, p<0.001$; respectively). The decrease observed between the control group and the Age2 group also reached a significant level $(45 \%, p<0.05)$. No other significant differences between groups were observed in the TO ratios in the striatum.

Statistical analysis of DA TO ratios in the prefrontal cortex revealed a significant effect of group $[F(3)=5.83, p<0.05 ; F(3)=6.17, p<0.05$; DOPAC/DA and (DOPAC + HVA)/DA respectively]. In the prefrontal cortex, the highest DOPAC/DA ratio was observed in the 12 months group even though not significantly differing from the control group. Both the MA and 20 months groups presented significantly lower TO ratios when compared with the 12 months group ( $45 \%, p<0.05$ and $56 \%, p<0.001)$. The lowest (DOPAC + HVA)/DA ratio was observed in the 20 months group. This value is significantly different from the control group $(43 \%, p<0.05)$ and the 12 months group $(46 \%, p<0.05)$. Statistical analysis of the 5 HT TO ratio in the prefrontal cortex revealed a significant effect of group $[F(3)=3.142, p=0.04]$. The $5 \mathrm{HIAA} / 5 \mathrm{HT}$ TO ratio was significantly different between the control group and the 20 months group $(35 \%, p=0.031)$. No further significant differences were observed.

None of the TO ratios calculated with reference to the hippocampus presented statistical differences between the studied groups. 


\section{Discussion}

In the present study we investigated the memory performance of Wistar rats repeatedly exposed to MA, when compared with both an age-matched control group and with chronologically aged animals. Additionally, we determined the levels of monoamines and their metabolites in the prefrontal cortex, hippocampus and the striatum, brain regions known to be involved in the memory function. The key findings are that repeated exposure to MA results in a loss of long, but not short, time object recognition memory and a depletion of monoamines and their metabolites. The results closely matched those obtained in the aged animal groups.

We found that extended treatment with MA induced a general exploration increase when compared with both the age-matched control group and the aged animal groups. These results are in contrast with previous studies that reported, depending on the analyzed delay, either no change, or a decreased activity, in MAtreated animals (Boger et al., 2007 and Boger et al., 2009). The differences may relate to the different drug administration paradigm and also the different species used. The exploration reduction observed in the aged groups is, however, in agreement with a previous report (Bergado et al., 2011). According to the present study, when a new object is introduced after a 15 min delay in the arena, all animals equally engage themselves in exploration activities and are able to detect novelty, a finding supported by previous work (Bergado et al., 2011). Nevertheless, it is interesting to note that even though MA-treated animals and aged animals recognized novelty in the short time delay $(15 \mathrm{~min})$, when the period between the presentation of objects was increased ( $24 \mathrm{~h}$ ) these groups lost the ability to recognize different objects. Increasing the time between the presentation of the familiar and the new object rendered aged animals unable to recognize novelty (Luparini et al., 2000).

The initial behavior of the tested animals (first day of habituation) revealed an increased frequency of approaches to the object by the MA age-matched control group when compared with the MA and the aged groups, which may possibly be interpreted as a less neophobic behavior. This reduced behavior in MA and aged animals may also be related to an increased preference to move along the walls when exploring a new environment (thigmotaxis). The higher frequency of approaches to the object in the first exposure to the apparatus may manifest a less anxiogenic response by the control group when compared with both the MA group and the aged groups. This neophobic behavior may be linked to a previously reported MA-induced thigmotaxis and may also serve as a valid indication of an anxiety-like state (Camarasa et al., 2010 and Treit and Fundytus, 1988). Our findings concerning the performance of the aged animals in the first exposure to the arena are in agreement with reports of increased anxiety among aged rats in the elevated T-maze (Boguszewski and Zagrodzka, 2002).

It has previously been shown, both in animals and humans, that repeated MA exposure results in long-term functional changes to the dopaminergic and serotonergic systems (Chang et al., 2007 and Volz et al., 2007). Nevertheless, assessing the nature of these changes (neuronal damage versus compensatory adaptations) is not always straightforward. In the current study, levels of DA, 5HT and their metabolites were determined after a prolonged withdrawal from binge repeated MA administration. In agreement with early work (Wagner et al., 1980b), the MA exposure paradigm used in the present study profoundly altered the dopaminergic system in the striatum, with a consistent depletion of DA and related metabolites. Corroborating previous reports (Miguez et al., 1999 and Ossowska, 1993), we observed an aged-related decline of the same dopaminergic parameters. These alterations, which occur during the aging of the brain and also during agerelated neurodegenerative processes such as Parkinson's and Alzheimer's diseases, have been associated with MA misuse (Garwood et al., 2006 and Guilarte, 2001). Indeed, the cellular mechanisms of the DA precursor L-3,4-dihydroxyphenylalanine (L-DOPA), which is the classic therapy used in Parkinson's disease, shows major similarities with MA (Biagioni et al., 2009). 
Changes in DOPAC, HVA or TO ratios have been used to estimate dopaminergic transmission, with lower production of DOPAC and HVA and reduced TO indicating reduced transmission. In the present study, the HVA:DA ratio was consistently reduced in both the MA and aged groups when compared to the control group. Previous studies, using extended MA self-administration in rats, have also reported a reduced dopaminergic activity, which occurs simultaneously with DA transporter down regulation (Schwendt et al., 2009). The (DOPAC + HVA)/DA ratio was only impaired in an age-dependent manner. These results are in agreement with previous studies that reported a decrease in the striatal DA TO in aged rats (Carfagna et al., 1985, Ponzio et al., 1978 and Venero et al., 1991). The well-known loss of dopaminergic neurons that project to the striatum may be linked to the above mentioned scenario (Kish et al., 1992). Indeed, the DA decrease observed during aging may relate to the decrease in tyrosine hydroxylase activity, with downstream repercussions at the level of DA synthesis (Reis et al., 1977). Similar consequences result from repeated MA exposure (Frost and Cadet, 2000, Harvey et al., 2000, Seiden et al., 1993, Sekine et al., 2001 and Volkow et al., 2001).

The hippocampus receives dopaminergic innervations mainly through the mesolimbic tract, which originates in the ventrotegmental region (Gasbarri et al., 1994). Previously, it has been suggested that hippocampal and striatal DA contents are similarly affected by age, while in other brain regions such as the prefrontal cortex, the dopaminergic content remains unchanged (Miguez et al., 1999). In the present work, we too report an age-related impairment in DA content in the hippocampus, but not in the prefrontal cortex. In the prefrontal cortex, we only observed an age-related decline in the levels of DOPAC and DA TO. It is important to note that we found similar results for the aged and MA groups concerning DA. Previous data support these findings; indeed, the relation between MA neurotoxicity and DA impairment in different brain regions has long been established (Friedman et al., 1998 and Sabol et al., 2001).

MA also affects the serotonergic system. The first reports assessing this issue noted significant depletion of $5 \mathrm{HT}$, with an associated loss of $5 \mathrm{HT}$ transporters, 3 weeks after the cessation of treatment (Ricaurte et al., 1980), and a reduction in tryptophan hydroxylase activity in the striatum and hippocampus after 1 month of the MA treatment (Hotchkiss and Gibb, 1980). In our work, MA-related 5HT contents and respective TO were only impaired in the hippocampus, not the striatum. With respect to the age-related alterations in the serotonergic system, the published literature is inconsistent. There are reports of unchanged (Moretti et al., 1987, Ponzio et al., 1982 and Pradhan, 1980), increased (Santiago et al., 1988 and Venero et al., 1993), or decreased (Venero et al., 1991) 5HT content and 5HT TO ratio. Concerning the 5HT content, our results show an age-related impairment in the prefrontal cortex. The 5HIAA content presented an age-related impairment in the hippocampus. 5HT TO was elevated in older animals, which is in agreement with previous data (Miguez et al., 1999), and may reflect an age-dependent increase in oxidative deamination occurring in the $5 \mathrm{HT}$ terminal areas in opposition to a lower synthesis of the amine.

Recent studies have correlated striatum, prefrontal cortex and hippocampus dopamine and serotonin deficits produced either by a repeated MA exposure (Lu et al., 2010) or observed in aged (20 months) rats (Esteban et al., 2010a and Esteban et al., 2010b) with cognitive deficits, corroborating the data presented here. Indeed, Lu and colleagues report an impairment of DA in the prefrontal cortex and an impairment in 5HT in the hippocampus in male mice with cognitive deficits (Lu et al., 2010). Esteban and colleagues also observed deficits in DA, $5 \mathrm{HT}$ and metabolites in the striatum and hippocampus in aged rats with cognitive impairments (Esteban et al., 2010a).

\section{Conclusion}

MA chronic exposure has been previously linked with schizophrenia's recognition memory deficits (Simon et al., 2000) and has been used to model this condition, overall, the present set of data also demonstrates that MA administration largely mimics the complex effects of chronological aging on the neurotransmitter 
systems, with similar alterations in object recognition memory. The striatum, prefrontal cortex and hippocampus are target brain regions actively involved in both MA- and age-related neurodegenerative processes, including cognitive and learning impairments that may be associated with Dementia, Parkinson's and Alzheimer's diseases. Understandably, research in this field has major social and medical relevance. MA-induced "accelerated aging" would increase the burden of elderly care. The present work contributes to the development of a feasible animal model to study the neuronal processes associated with aging.

\section{References}

Ali et al., 1993 S.F. Ali, S.N. David, G.D. Newport Age-related susceptibility to MPTP-induced neurotoxicity in mice Neurotoxicology, 14 (1993), pp. 29-34

Alves et al., 2009 E. Alves, Z. Binienda, F. Carvalho, C.J. Alves, E. Fernandes, M.D.L. Bastos, et al. AcetylL-carnitine provides effective in vivo neuroprotection over 3,4-methylenedioximethamphetamineinduced mitochondrial neurotoxicity in the adolescent rat brain Neuroscience, 158 (2009), pp. 514523

Belcher et al., 2006 A.M. Belcher, S.J. O'Dell, J.F. Marshall A sensitizing regimen of methamphetamine causes impairments in a novelty preference task of object recognition Behav Brain Res, 170 (2006), pp. 167-172

Belcher et al., 2008 A.M. Belcher, E.M. Feinstein, S.J. O'Dell, J.F. Marshall Methamphetamine influences on recognition memory: comparison of escalating and single-day dosing regimens Neuropsychopharmacology, 33 (2008), pp. 1453-1463

Bergado et al., 2011 J.A. Bergado, W. Almaguer, Y. Rojas, V. Capdevila, J.U. Frey Spatial and emotional memory in aged rats: a behavioral-statistical analysis Neuroscience, 172 (2011), pp. 256-269

Biagioni et al., 2009 F. Biagioni, A. Pellegrini, S. Ruggieri, L. Murri, A. Paparelli, F. Fornai Behavioural sensitisation during dopamine replacement therapy in Parkinson's disease is reminiscent of the addicted brain Curr Top Med Chem, 9 (2009), pp. 894-902

Bisagno et al., 2002 V. Bisagno, D. Ferguson, V.N. Luine Short toxic methamphetamine schedule impairs object recognition task in male rats Brain Res, 940 (2002), pp. 95-101

Boger et al., 2007 H.A. Boger, L.D. Middaugh, K.S. Patrick, S. Ramamoorthy, E.D. Denehy, H. Zhu, et al. Long-term consequences of methamphetamine exposure in young adults are exacerbated in glial cell line-derived neurotrophic factor heterozygous mice J Neurosci, 27 (2007), pp. 8816-8825

Boger et al., 2009 H.A. Boger, L.D. Middaugh, A.C. Granholm, J.F. McGinty Minocycline restores striatal tyrosine hydroxylase in GDNF heterozygous mice but not in methamphetamine-treated mice Neurobiol Dis, 33 (2009), pp. 459-466

Boguszewski and Zagrodzka, 2002 P. Boguszewski, J. Zagrodzka Emotional changes related to age in rats - a behavioral analysis Behav Brain Res, 133 (2002), pp. 323-332

Bubenikova-Valesova et al., 2009 V. Bubenikova-Valesova, P. Kacer, K. Syslova, L. Rambousek, M. Janovsky, B. Schutova, et al. Prenatal methamphetamine exposure affects the mesolimbic dopaminergic system and behavior in adult offspring Int J Dev Neurosci, 27 (2009), pp. 525-530 Camarasa et al., 2010 J. Camarasa, T. Rodrigo, D. Pubill, E. Escubedo Memantine is a useful drug to prevent the spatial and non-spatial memory deficits induced by methamphetamine in rats Pharmacol Res, 62 (2010), pp. 450-456

Capela et al., 2009 J.P. Capela, H. Carmo, F. Remiao, M.L. Bastos, A. Meisel, F. Carvalho Molecular and cellular mechanisms of ecstasy-induced neurotoxicity: an overview Mol Neurobiol, 39 (2009), pp. 210271 
Carfagna et al., 1985 N. Carfagna, F. Trunzo, A. Moretti Brain catecholamine content and turnover in aging rats Exp Gerontol, 20 (1985), pp. 265-269

Carnes et al., 2008 B.A. Carnes, D.O. Staats, W.E. Sonntag Does senescence give rise to disease? Mech Ageing Dev, 129 (2008), pp. 693-699

Carvalho, 2009 F. Carvalho How bad is accelerated senescence in consumers of drugs of abuse? Adicciones, 21 (2009), pp. 99-104

Chang et al., 2007 L. Chang, D. Alicata, T. Ernst, N. Volkow Structural and metabolic brain changes in the striatum associated with methamphetamine abuse Addiction, 102 (Suppl. 1) (2007), pp. 16-32

Cretzmeyer et al., 2003 M. Cretzmeyer, M.V. Sarrazin, D.L. Huber, R.I. Block, J.A. Hall Treatment of methamphetamine abuse: research findings and clinical directions J Subst Abuse Treat, 24 (2003), pp. 267-277

Cubells et al., 1994 J.F. Cubells, S. Rayport, G. Rajendran, D. Sulzer Methamphetamine neurotoxicity involves vacuolation of endocytic organelles and dopamine-dependent intracellular oxidative stress J Neurosci, 14 (1994), pp. 2260-2271

Dowling et al., 2008 G.J. Dowling, S.R.B. Weiss, T.P. Condon Drugs of abuse and the aging brain Neuropsychopharmacology, 33 (2008), pp. 209-218

Eisch et al., 1998 A.J. Eisch, L.C. Schmued, J.F. Marshall Characterizing cortical neuron injury with Fluoro-Jade labeling after a neurotoxic regimen of methamphetamine Synapse, 30 (1998), pp. 329333

Ennaceur et al., 1997 A. Ennaceur, N. Neave, J.P. Aggleton Spontaneous object recognition and object location memory in rats: the effects of lesions in the cingulate cortices, the medial prefrontal cortex, the cingulum bundle and the fornix Exp Brain Res, 113 (1997), pp. 509-519

Ennaceur et al., 2005 A. Ennaceur, S. Michalikova, A. Bradford, S. Ahmed Detailed analysis of the behavior of Lister and Wistar rats in anxiety, object recognition and object location tasks Behav Brain Res, 159 (2005), pp. 247-266

Erickson and Barnes, 2003 C.A. Erickson, C.A. Barnes The neurobiology of memory changes in normal aging Exp Gerontol, 38 (2003), pp. 61-69

Esteban et al., 2010a S. Esteban, C. Garau, S. Aparicio, D. Moranta, P. Barcelo, M.A. Fiol, et al. Chronic melatonin treatment and its precursor L-tryptophan improve the monoaminergic neurotransmission and related behavior in the aged rat brain J Pineal Res, 48 (2010), pp. 170-177

Esteban et al., 2010b S. Esteban, C. Garau, S. Aparicio, D. Moranta, P. Barcelo, M. Ramis, et al. Improving effects of long-term growth hormone treatment on monoaminergic neurotransmission and related behavioral tests in aged rats Rejuvenation Res, 13 (2010), pp. 707-716

Fleckenstein et al., 1997 A.E. Fleckenstein, R.R. Metzger, D.G. Wilkins, J.W. Gibb, G.R. Hanson Rapid and reversible effects of methamphetamine on dopamine transporters J Pharmacol Exp Ther, 282 (1997), pp. 834-838

Friedman et al., 1998 S.D. Friedman, E. Castaneda, G.K. Hodge Long-term monoamine depletion, differential recovery, and subtle behavioral impairment following methamphetamine-induced neurotoxicity Pharmacol Biochem Behav, 61 (1998), pp. 35-44

Frost and Cadet, 2000 D.O. Frost, J.L. Cadet Effects of methamphetamine-induced neurotoxicity on the development of neural circuitry: a hypothesis Brain Res Brain Res Rev, 34 (2000), pp. 103-118 Fumagalli et al., 1999 F. Fumagalli, R.R. Gainetdinov, Y.M. Wang, K.J. Valenzano, G.W. Miller, M.G. Caron Increased methamphetamine neurotoxicity in heterozygous vesicular monoamine transporter 2 knock-out mice J Neurosci, 19 (1999), pp. 2424-2431

Garwood et al., 2006 E.R. Garwood, W. Bekele, C.E. McCulloch, C.W. Christine Amphetamine exposure is elevated in Parkinson's disease Neurotoxicology, 27 (2006), pp. 1003-1006 
Gasbarri et al., 1994 A. Gasbarri, C. Verney, R. Innocenzi, E. Campana, C. Pacitti Mesolimbic dopaminergic neurons innervating the hippocampal formation in the rat: a combined retrograde tracing and immunohistochemical study Brain Res, 668 (1994), pp. 71-79

Graham, 1978 D.G. Graham Oxidative pathways for catecholamines in the genesis of neuromelanin and cytotoxic quinones Mol Pharmacol, 14 (1978), pp. 633-643

Guilarte, 2001 T.R. Guilarte Is methamphetamine abuse a risk factor in parkinsonism? Neurotoxicology, 22 (2001), pp. 725-731

Harvey et al., 2000 D.C. Harvey, G. Lacan, S.P. Tanious, W.P. Melega Recovery from methamphetamine induced long-term nigrostriatal dopaminergic deficits without substantia nigra cell loss Brain Res, 871 (2000), pp. 259-270

Hotchkiss and Gibb, 1980 A.J. Hotchkiss, J.W. Gibb Long-term effects of multiple doses of methamphetamine on tryptophan hydroxylase and tyrosine hydroxylase activity in rat brain $\mathrm{J}$ Pharmacol Exp Ther, 214 (1980), pp. 257-262

Jones et al., 1998 S.R. Jones, R.R. Gainetdinov, R.M. Wightman, M.G. Caron Mechanisms of amphetamine action revealed in mice lacking the dopamine transporter J Neurosci, 18 (1998), pp. 1979-1986

Kalechstein et al., 2003 A.D. Kalechstein, T.F. Newton, M. Green Methamphetamine dependence is associated with neurocognitive impairment in the initial phases of abstinence $\mathrm{J}$ Neuropsychiatry Clin Neurosci, 15 (2003), pp. 215-220

Kish et al., 1992 S.J. Kish, K. Shannak, A. Rajput, J.H. Deck, O. Hornykiewicz Aging produces a specific pattern of striatal dopamine loss: implications for the etiology of idiopathic Parkinson's disease $\mathrm{J}$ Neurochem, 58 (1992), pp. 642-648

Koda and Gibb, 1973 L.Y. Koda, J.W. Gibb Adrenal and striatal tyrosine hydroxylase activity after methamphetamine J Pharmacol Exp Ther, 185 (1973), pp. 42-48

Lu et al., 2010 P. Lu, T. Mamiya, L.L. Lu, A. Mouri, M. Niwa, H.C. Kim, et al. Silibinin attenuates cognitive deficits and decreases of dopamine and serotonin induced by repeated methamphetamine treatment Behav Brain Res, 207 (2010), pp. 387-393

Luine et al., 1990 V. Luine, D. Bowling, M. Hearns Spatial memory deficits in aged rats - contributions of monoaminergic systems Brain Res, 537 (1990), pp. 271-278

Luparini et al., 2000 M.R. Luparini, A. Del Vecchio, G. Barillari, M. Magnani, M. Prosdocimi Cognitive impairment in old rats: a comparison of object displacement, object recognition and water maze Aging (Milano), 12 (2000), pp. 264-273

Marshall et al., 2007 J.F. Marshall, A.M. Belcher, E.M. Feinstein, S.J. O'Dell Methamphetamine-induced neural and cognitive changes in rodents Addiction, 102 (2007), pp. 61-69

Melega et al., 1995 W.P. Melega, A.E. Williams, D.A. Schmitz, E.W. DiStefano, A.K. Cho Pharmacokinetic and pharmacodynamic analysis of the actions of D-amphetamine and D-methamphetamine on the dopamine terminal J Pharmacol Exp Ther, 274 (1995), pp. 90-96

Melo et al., 2010 P. Melo, V. Zanon-Moreno, C.J. Alves, A. Magalhaes, M.A. Tavares, M.D. Pinazo-Duran, et al. Oxidative stress response in the adult rat retina and plasma after repeated administration of methamphetamine Neurochem Int, 56 (2010), pp. 431-436

Meredith et al., 2005 C.W. Meredith, C. Jaffe, K. Ang-Lee, A.J. Saxon Implications of chronic methamphetamine use: a literature review Harv Rev Psychiatry, 13 (2005), pp. 141-154

Metzger et al., 2000 R.R. Metzger, H.M. Haughey, D.G. Wilkins, J.W. Gibb, G.R. Hanson, A.E. Fleckenstein Methamphetamine-induced rapid decrease in dopamine transporter function: role of dopamine and hyperthermia J Pharmacol Exp Ther, 295 (2000), pp. 1077-1085 
Miguez et al., 1999 J.M. Miguez, M. Aldegunde, L. Paz-Valinas, J. Recio, E. Sanchez-Barcelo Selective changes in the contents of noradrenaline, dopamine and serotonin in rat brain areas during aging $\mathrm{J}$ Neural Transm, 106 (1999), pp. 1089-1098

Moretti et al., 1987 A. Moretti, N. Carfagna, F. Trunzo Effect of aging on monoamines and their metabolites in the rat brain Neurochem Res, 12 (1987), pp. 1035-1039

Nagai et al., 2007 T. Nagai, K. Takuma, M. Dohniwa, D. Ibi, H. Mizoguchi, H. Kamei, et al. Repeated methamphetamine treatment impairs spatial working memory in rats: reversal by clozapine but not haloperidol Psychopharmacology, 194 (2007), pp. 21-32

Nash and Yamamoto, 1992 J.F. Nash, B.K. Yamamoto Methamphetamine neurotoxicity and striatal glutamate release: comparison to 3,4-methylenedioxymethamphetamine Brain Res, 581 (1992), pp. 237-243

Nordahl et al., 2003 T.E. Nordahl, R. Salo, M. Leamon Neuropsychological effects of chronic methamphetamine use on neurotransmitters and cognition. A review J Neuropsychiatry Clin Neurosci, 15 (2003), pp. 317-325

Ossowska, 1993 K. Ossowska Disturbances in neurotransmission processes in aging and age-related diseases Pol J Pharmacol, 45 (1993), pp. 109-131

Ponzio et al., 1978 F. Ponzio, N. Brunello, S. Algeri Catecholamine synthesis in brain of ageing rat $\mathrm{J}$ Neurochem, 30 (1978), pp. 1617-1620

Ponzio et al., 1982 F. Ponzio, G. Calderini, G. Lomuscio, G. Vantini, G. Toffano, S. Algeri Changes in monoamines and their metabolite levels in some brain regions of aged rats Neurobiol Aging, 3 (1982), pp. 23-29

Pradhan, 1980 S.N. Pradhan Central neurotransmitters and aging Life Sci, 26 (1980), pp. 1643-1656

Reis et al., 1977 D.J. Reis, R.A. Ross, T.H. Joh Changes in the activity and amounts of enzymes synthesizing catecholamines and acetylcholine in brain, adrenal medulla, and sympathetic ganglia of aged rat and mouse Brain Res, 136 (1977), pp. 465-474

Ricaurte et al., 1980 G.A. Ricaurte, C.R. Schuster, L.S. Seiden Long-term effects of repeated methylamphetamine administration on dopamine and serotonin neurons in the rat brain: a regional study Brain Res, 193 (1980), pp. 153-163

Ricaurte et al., 1982 G.A. Ricaurte, R.W. Guillery, L.S. Seiden, C.R. Schuster, R.Y. Moore Dopamine nerve terminal degeneration produced by high doses of methylamphetamine in the rat brain Brain Res, 235 (1982), pp. 93-103

Ricaurte et al., 1983a G.A. Ricaurte, R.W. Fuller, K.W. Perry, L.S. Seiden, C.R. Schuster Fluoxetine increases long-lasting neostriatal dopamine depletion after administration of D-methamphetamine and D-amphetamine Neuropharmacology, 22 (1983), pp. 1165-1169

Ricaurte et al., 1983b G.A. Ricaurte, L.S. Seiden, C.R. Schuster Increased dopamine metabolism in the rat neostriatum after toxic doses of D-methylamphetamine Neuropharmacology, 22 (1983), pp. 13831388

Robinson et al., 1990 T.E. Robinson, J. Yew, P.E. Paulson, D.M. Camp The long-term effects of neurotoxic doses of methamphetamine on the extracellular concentration of dopamine measured with microdialysis in striatum Neurosci Lett, 110 (1990), pp. 193-198

Rogers et al., 2008 J.L. Rogers, S. De Santis, R.E. See Extended methamphetamine self-administration enhances reinstatement of drug seeking and impairs novel object recognition in rats Psychopharmacology (Berl), 199 (2008), pp. 615-624 
Sabol et al., 2001 K.E. Sabol, J.T. Roach, S.L. Broom, C. Ferreira, M.M. Preau Long-term effects of a high-dose methamphetamine regimen on subsequent methamphetamine-induced dopamine release in vivo Brain Res, 892 (2001), pp. 122-129

Salo et al., 2009 R. Salo, S. Ursu, M.H. Buonocore, M.H. Leamon, C. Carter Impaired prefrontal cortical function and disrupted adaptive cognitive control in methamphetamine abusers: a functional magnetic resonance imaging study Biol Psychiatry, 65 (2009), pp. 706-709

Santiago et al., 1988 M. Santiago, A. Machado, F. Reinoso-Suarez, J. Cano Changes in biogenic amines in rat hippocampus during development and aging Life Sci, 42 (1988), pp. 2503-2508

Schroder et al., 2003 N. Schroder, S.J. O'Dell, J.F. Marshall Neurotoxic methamphetamine regimen severely impairs recognition memory in rats Synapse, 49 (2003), pp. 89-96

Schwendt et al., 2009 M. Schwendt, A. Rocha, R.E. See, A.M. Pacchioni, J.F. McGinty, P.W. Kalivas Extended methamphetamine self-administration in rats results in a selective reduction of dopamine transporter levels in the prefrontal cortex and dorsal striatum not accompanied by marked monoaminergic depletion J Pharmacol Exp Ther, 331 (2009), pp. 555-562

Scott et al., 2007 J.C. Scott, S.P. Woods, G.E. Matt, R.A. Meyer, R.K. Heaton, J.H. Atkinson, et al. Neurocognitive effects of methamphetamine: a critical review and meta-analysis Neuropsychol Rev, 17 (2007), pp. 275-297

Seiden et al., 1993 L.S. Seiden, K.E. Sabol, G.A. Ricaurte Amphetamine: effects on catecholamine systems and behavior Annu Rev Pharmacol Toxicol, 33 (1993), pp. 639-677

Sekine et al., 2001 Y. Sekine, M. lyo, Y. Ouchi, T. Matsunaga, H. Tsukada, H. Okada, et al. Methamphetamine-related psychiatric symptoms and reduced brain dopamine transporters studied with PET Am J Psychiatry, 158 (2001), pp. 1206-1214

Simon et al., 2000 S.L. Simon, C. Domier, J. Carnell, P. Brethen, R. Rawson, W. Ling Cognitive impairment in individuals currently using methamphetamine Am J Addict, 9 (2000), pp. 222-231

Simon et al., 2004 S.L. Simon, J. Dacey, S. Glynn, R. Rawson, W. Ling The effect of relapse on cognition in abstinent methamphetamine abusers J Subst Abuse Treat, 27 (2004), pp. 59-66

Treit and Fundytus, 1988 D. Treit, M. Fundytus Thigmotaxis as a test for anxiolytic activity in rats Pharmacol Biochem Behav, 31 (1988), pp. 959-962

Troen, 2003 B.R. Troen The biology of aging Mt Sinai J Med, 70 (2003), pp. 3-22

Venero et al., 1991 J.L. Venero, A. Machado, J. Cano Turnover of dopamine and serotonin and their metabolites in the striatum of aged rats J Neurochem, 56 (1991), pp. 1940-1948

Venero et al., 1993 J.L. Venero, C. de la Roza, A. Machado, J. Cano Age-related changes on monoamine turnover in hippocampus of rats Brain Res, 631 (1993), pp. 89-96

Volkow et al., 2001 N.D. Volkow, L. Chang, G.J. Wang, J.S. Fowler, M. Leonido-Yee, D. Franceschi, et al. Association of dopamine transporter reduction with psychomotor impairment in methamphetamine abusers Am J Psychiatry, 158 (2001), pp. 377-382

Volz et al., 2007 T.J. Volz, A.E. Fleckenstein, G.R. Hanson Methamphetamine-induced alterations in monoamine transport: implications for neurotoxicity, neuroprotection and treatment Addiction, 102 (Suppl. 1) (2007), pp. 44-48

Vorhees et al., 2005 C.V. Vorhees, T.M. Reed, L.L. Morford, M. Fukumura, S.L. Wood, C.A. Brown, et al. Periadolescent rats (P41-50) exhibit increased susceptibility to D-methamphetamine-induced longterm spatial and sequential learning deficits compared to juvenile (P21-30 or P31-40) or adult rats (P51-60) Neurotoxicol Teratol, 27 (2005), pp. 117-134

Vupputuri et al., 2004 S. Vupputuri, V. Batuman, P. Muntner, L.A. Bazzano, J.J. Lefante, P.K. Whelton, et al. Risk for mild kidney function decline associated with illicit drug use among hypertensive men Am $J$ Kidney Dis, 43 (2004), pp. 629-635 
Wagner et al., 1980a G.C. Wagner, G.A. Ricaurte, C.E. Johanson, C.R. Schuster, L.S. Seiden Amphetamine induces depletion of dopamine and loss of dopamine uptake sites in caudate Neurology, 30 (1980), pp. 547-550

Wagner et al., 1980b G.C. Wagner, G.A. Ricaurte, L.S. Seiden, C.R. Schuster, R.J. Miller, J. Westley Longlasting depletions of striatal dopamine and loss of dopamine uptake sites following repeated administration of methamphetamine Brain Res, 181 (1980), pp. 151-160

White et al., 2009 I.M. White, T. Minamoto, J.R. Odell, J. Mayhorn, W. White Brief exposure to methamphetamine (METH) and phencyclidine (PCP) during late development leads to long-term learning deficits in rats Brain Res, 1266 (2009), pp. 72-86 